\section{ENDOMETRIAL CANCER IN CLINICAL PRACTICE: THE EXPERIENCE OF AN ONCOLOGICAL CENTER}

R Fontes*, J Rodrigues, C Oliveira, M Peixoto, F Pereira, J Cunha, C Portela, A Marques, R Nabiço, L Queiroz. Portugal, Medical Oncology, Portugal

10.1136/ijgc-2021-ESG0.148

Introduction/Background* Endometrial cancer is the fourth most common malignancy among women and the most frequent gyneacological cancer in developed countries. Generally it is associated with a favourable prognosis mainly because most cases are diagnosed at an early stage. This work aims to evaluate the real-life data of an oncological center.

Methodology Retrospective analysis of data from patients diagnosed and treated in our institution, from January 2009 to December 2019.

Result(s)* During this period, 217 patients were diagnosed with endometrial cancer, with a median age at diagnosis of 67 years. Regarding clinical staging, the majority of them were in early stage disease - FIGO I/II $(69 \%, n=149)$ and mainly endometrioid histological subtype $(71 \%, \mathrm{n}=154)$.

High-risk endometrial cancer was seen in $41,9 \%$ of the patients $(n=91)$. From these, 36,3\% $(n=33)$ were treated with adjuvant radiotherapy, with or without braquitherapy, and $56 \%(n=51)$ also received chemotherapy (carboplatin + paclitaxel) before radiation treatment. Recorrence occured in 23 patients, mainly FIGO stage IIIC1.

Overall, relapse was detected in 36 patients (16,6\%) - 15 patients with loco-regional disease and 21 with distant metastasis - with a median progression free survival of 12 months. Histology was found to be the major risk factor $(p=0,05)$ for recorrence.

To date, the number of deaths was $64(29,5 \%)$, but 15 patients died from other causes. In multivariate analysis, the independent prognostic factos were histology $(p=0,01)$, FIGO stage $(p=0,05)$ and limphovascular involvement $(p=0,00)$, with the last one being the most significant $(B=1,077)$. Overall survival was $82,3 \%$ and $72,2 \%$ at 2 and 5 years, respectively.

From the 217 patients 61,8\% $(n=133)$ are under followup, showing no signs of recorrence.

Conclusion* Although endometrial cancer is generaly diagnosed at an early stage, differences in patient characteristics and histopathological features of the disease impact on both prognosis and treatment approach. Over the past few decades, several studies have demonstrated the prognostic importance of different parameters including histological subtype and grade, stage of disease and lymphovascular involvement. Taking into account the advances in the oncology setting, the future will probably be focused on the molecular profile of endometrial cancer.

\section{EXTERNAL VALIDATION OF THE NORWICH DEFAB SCORE FOR UP-FRONT PREDICTION OF ENDOMETRIAL CANCER}

J Davies, K Hartshorn, L Honeyman, S Kolhe, A Phillips. Derby Gynaecological Cancer Centre, derby, UK

10.1136/ijgc-2021-ESGO.149
Introduction/Background* The DEFAB score combines clinical data and patient characteristics such as Age, endometrial thickness, diabetes, BMI and bleeding characteristics to calculate the risk of endometrial cancer causing PMB. It was based on 3047 patients cared for between 2006 and 2009. Inclusion was all women presenting with PMB. The original authors proposed a cut off score of 3 - whereby a score of $\leq 2$ is managed based on ultrasound assessment of endometrial thickness; whereas a score of $\geq 3$ was additionally investigated with endometrial biopsy regardless of any ultrasound findings, and proceeded to hysteroscopy if this biopsy is negative.

Methodology We compared the baseline characteristics of our cohort to the original internal validation study and retrospectively applied the DEFAB score to our patient group to determine how it performed.

Result(s)* Mean age was 61 (50-95) years, BMI was 30.7 (25-36) and $30.2(25-36)$ in cancer and non-cancer patients. Diabetes was present in $20(20.6 \%)$ and 93 (9.7\%) cancer and non-cancer patients. The bleeding pattern was recurrent in $39(40 \%)$, and $309(32.2 \%)$ in cancer and non-cancer patients.

42 cancer patients had a DEFAB score of $\leq 2$, of whom 41 met the endometrial thickness criteria and went on to have a cancer diagnosed. There was one cancer found in a patient who the DEFAB protocol would have discharged without diagnosis, a false negative rate of $2.4 \%$.

The incidence of cancer in patients with a DEFAB score of $>3(\mathrm{n}=457)$, was $58(12.7 \%$.). The sensitivity of the DEFAB \& ET thresholding protocol was $98.7 \%$ if the cut-off DEFAB score of 3 is used, with a specificity of $60.1 \%$.

Conclusion* The DEFAB score performs well at the proposed cutoff in our cohort.

\section{DEEP INFILTRATING ENDOMETRIOSIS: NEOPLASM OR BENIGN CONDITION?}

K Grammatikakis*, A Theodoropoulou, E Halkia, K Patsouras. Tzaneio Prefecture General Hospital of Piraeus, Pireas, Greece

\subsection{6/ijgc-2021-ESGO.150}

Introduction/Background* Deep infiltrating endometriosis is an interesting rare subtype of endometriosis which was recently subjected to genomic evaluation. There are three major subtypes of endometriosis up to date, ovarian endometrioma (OMA), superficial peritoneal endometriosis (SPE), and deep infiltrating endometriosis (DIE).Deep endometriosis has the particular nature to locally invade surrounding structures such as bowel, bladder and ureter) but rarely metastasizes. The present knowledge indicates that hormonal function and immunological factors, such as peritoneal macrophages, natural killer cells, and lymphocytes, are sensibly altered in DIE.Invasive mechanisms are more expressed in DIE in comparison to the OMA and SPE. Correlated with the increased invasiveness are the data on very high expression of neuroangiogenesis genes in DIE.

Methodology Our aim was to evaluate the latest data that can correlate the microenvironment of deep infiltrating endometriosis (DIE) with the one of endometrial cancer, discuss the possibility that perhaps even the deep-infiltrating subtype of 